\title{
Tracking Sleep Times to Reduce Tiredness and Improve Sleep in College Students
}

\author{
Daniel J. Prestwich, Linda L. Rankin, and Jeff Housman \\ Idaho State University
}

\begin{abstract}
The purpose of this study was to determine the effect that additional sleep health education, as well as recording amounts of nightly sleep had on the amounts of sleep and sense of daytime sleepiness, experienced by participants enrolled in a personal health class at a state university in the Pacific Northwest. Participants were divided into four groups, a control, a group that received sleep education only, a group that kept a sleep log only, and a group that received both interventions. Outcomes were assessed with a three day sleep recall, and using the Epworth Sleepiness Scale (ESS). The students who received both interventions improved their sleep by over 50 minutes per night.
\end{abstract}

(C) 2007 Californian Journal of Health Promotion. All rights reserved.

Keywords: college students, sleep, logging, journaling

\section{Introduction}

Research indicates that many college students are sleeping too few hours $(6.85+/-1.04)$, and many report poor quality of sleep (Hicks, Hernandez \& Pellegrini, 2001a). Variables such as availability of academic resources (e.g.. library hours), roommates, class schedules, and sleep hygiene may affect the amount and quality of sleep among college students, and these factors may affect how and when students work, play and study. Furthermore, too little sleep and poor sleep quality may affect overall health and academic performance. An international study done by Steptoe, Peacey and Wardle (2006), showed a correlation between reduced sleep times and low scores on a self-rated overall health scale among university students. Howell, Jahrig and Powell (2004) suggest that those who carry a full academic schedule, as well as those who take more demanding courses, are more likely to have a lower GPA when sleep problems are present.

Engle-Friedman et al. (2003) devised a study where half of the subjects lost a prior night's sleep, and half did not. The following day, the subjects were invited to choose sets of math problems to complete. The subjects who were sleep deprived tended to choose easier sets of problems, even when instructed to give their best effort. The percentages of questions answered right and wrong were similar in the two groups, but the sleep deprived subjects chose to answer easier questions. The authors suggest that being sleep deprived contributes to individuals choosing "low-effort" behavior which allows them to perform with success, but at a lower skill level. College participants themselves often identify problems with their own sleep patterns. Hicks, Fernandez, and Pellegrini (2001b) showed that $71 \%$ of the college students who participated in their study expressed dissatisfaction with their sleep quality. This was an increase from $24 \%$ in 1978 , and $53 \%$ in 1988 , as demonstrated in previous studies by the authors. A study by Coren (1994) showed that $64 \%$ of his subjects ages $17-30$ had sleep difficulties. This result was replicated by Buboltz, Brown, and Soper (2001) who explained that $73 \%$ of their college age respondents reported unsatisfactory sleep amounts and or quality. Conversely, the authors found that only $11 \%$ of their college student respondents could be put in the category of meeting sleep needs consistently.

An emerging debate on college campuses relates to the closing time of college resources, such as libraries and computer centers (Capriccioso, 2002). Students' schedules seem to be shifting to 
times later in the evening (and morning), and many feel that a closing time of midnight is dated and unrealistic for life in the modern era. On the other hand, administrators express concern that keeping facilities open until three or four in the morning may be enabling students to be undisciplined in their schedules and to habitually rely on caffeine and energy drinks to make up for lost sleep.

A significant health issue among college students is depression, which multiple researchers have demonstrated is associated with sleep deprivation (Alapin et al., 2000; Allgower, Wardle, and Steptoe, 2001; Dennis \& Ross, 2005; Giesecke, 1987; Jindal \& Thase, 2004; Meltzer \& Mindell, 2007; Peppard, Szklo-Coxe, Hla, and Young, 2006; Pilcher, Ginter, and Sadowsky, 1997; Steptoe, Peacey, and Wardle, 2006). There is, however, a lack of determined causality.

Goldberg, Shah, Halstead, and McNamara (1999) studied recent victims of unintentional trauma in the emergency room to learn what portion of them were sleep deprived prior to their injury. The authors learned that many forms of acute and chronic sleep problems were often present in the injured respondents. Forty seven percent of respondents were chronically sleep deprived, especially during weekdays. The authors also found that often, immediately prior to the injury, there was an even greater reduction in sleep. A similar study was done by Edmonds and Vinson (2007). These authors suggest that those who have gotten seven days worth of good sleep are less likely to be injured.

A relatively new area of sleep research has been to correlate decreased sleep times with increased body mass index (BMI), as in Kohatsu et al. (2006). They studied a rural population in the United States, and reported that those who slept seven hours or more a night had a mean BMI below 30 (7-7.9 hours $=29.14,8-8.9$ hours $=$ 29.27, $\geq 9$ hours $=28.25$ ). The mean BMI of those who slept fewer than seven hours was greater than $30(6-6.9$ hours $=30.17 ;<6$ hours $=$ 30.24). Obesity is defined as a BMI of 30 or above and is predictive of future health outcomes.
Reduced sleep tends to predispose individuals to many and varied negative health and life outcomes. These include, but are not limited to depression, attention difficulties, generally lower mood states, affective strain, compromised motor skills and reflexive capacity, increased risk of involvement in an automobile crash, increased physical pain, poorer academic performance and outcomes, increased substance abuse, a false sense of confidence, and "low effort" behavior.

College students seem to be less satisfied with their sleep now than they have been in previous decades (Hicks, Hernandez, and Pellegrini, 2001b). This is additionally substantiated by the fact that they appear to be getting less sleep. Hicks \& Pellegrini (1991) completed a series of studies documenting sleep times of college age adults. Their research shows an hour decrease in median sleep times from 1969 (7.75 hours) to 1989 (6.75 hours), and Hicks, Hernandez, and Pellegrini (2001a) show that their 1,585 respondents had a mean sleep time of 6.85 hours (SD+/-1.04) (see Table 1). Lack (1986) expressed the view that chronic insomnia may be a modern occupational hazard of being enrolled in college.

The purpose of this study was to determine the effect that additional sleep health education, as well as recording amounts of sleep nightly had on the amount of sleep, and sense of daytime sleepiness, experienced by participants enrolled in a personal health class, Promoting Wellness (HE 200), at a state university in the Pacific Northwest during the spring semester, 2007.

\section{Methods}

All necessary approvals were obtained from the University's Institutional Review Board prior to the collection of any data. Participation was voluntary and consent forms were obtained from participants. No incentives were offered for participation.

\section{Research Design}

A pretest-posttest control group design was utilized, with two treatments, and four group variations, as described below. A convenience sample of students $(n=54)$ attending personal 
health classes during the spring 2007 semester participated in the study. There were five available sections, two of which were smaller than the others and were combined to form one group. Each of the four groups completed an Epworth Sleepiness Scale (ESS) survey, as well as a free recall of sleep received in the three days prior to the survey (Johns, 1991). Respondents also completed a demographics page. The groups were then randomly assigned as follows.

Table 1

Reduction in Sleep Times Among College Students Over Three Decades. Compiled from Hicks et al. (2001a) and Hicks and Pellegrini (1991)

\begin{tabular}{|c|c|}
\hline Year & Median Sleep Duration (hours/day) \\
\hline 1969 & 7.75 \\
\hline 1979 & 7.13 \\
\hline 1989 & 6.75 \\
\hline & \\
\hline & Mean Sleep Duration (hours/day +/- SD) \\
\hline 1989 & $6.87+/-1.02$ \\
\hline 2001 & $6.85+/-1.04$ \\
\hline
\end{tabular}

Table 2

Study Design by Group, Showing Diversities of Treatment

\begin{tabular}{|l|c|c|}
\hline Group & Sleep Education & Sleep Log \\
\hline A & No & No \\
\hline B & Yes & No \\
\hline C & No & Yes \\
\hline D & Yes & Yes \\
\hline
\end{tabular}

Group A. These participants served as the control group, and only completed the Epworth Sleepiness Scale, and three day sleep recall at the beginning and end of the research period.

Group B. These participants received an additional ten to fifteen minutes of sleep education following the initial survey. The sleep information given was based on information from the National Sleep Foundation (2005) regarding the concept of sleep debt. The most common effects of sleep deprivation were discussed, as well as some practical ideas for obtaining adequate sleep. Participants also completed a three day sleep recall and ESS survey at the end of the research period.

Group C. These participants did not receive additional sleep following the initial survey, but were given sleep logs to complete over the following two weeks. Participants also completed a three day sleep recall, and ESS survey at the end of the research.

Group D. These participants received both additional sleep education and kept a daily log of their sleep amounts over the two weeks. Participants also completed a three day sleep recall, and ESS survey at the end of the research. 


\section{Instrumentation}

After completing a demographics page, each participant completed a three day sleep recall as well as an ESS survey. A daily sleep log was then given to the members of groups $\mathrm{C}$ and $\mathrm{D}$ to track their sleep over the following two weeks. Each participant at the end of the research period again completed the three day sleep recall, as well as a final ESS.

The sleep recall simply asked participants to record sleep amounts (in hour and minutes) for the previous three nights, from memory. For the ESS, participants were asked to rate the level of sleepiness they believed would be experienced if placed in each of the eight commonly experienced settings, on a scale of 0-3.

The sleep logs themselves were used only by the participants of groups $\mathrm{C}$ and $\mathrm{D}$, and were not entered into the research findings as data. They were only used as a method of intervention to increase awareness of actual sleep. Because only half of the participants completed a log, there would be no comparison data across all groups to analyze.

\section{Participants}

The group consisted of mostly females $(n=38)$ and some males $(n=14)$. Of the 52 students, all but six were enrolled in at least 12 credits during the term, and each respondent was taking at least six credits. Most participants $(n=31)$ listed their activity level as "moderate", and the remainder were nearly perfectly split amongst those who considered their activity level "high" and "low". Most respondents reported being part-time workers. Only six of the subjects reported working more than 30 hours per week, and twelve had no outside school hours of work $($ Mean=17.5, S.D. $=13.28$, Median=20). Eleven participants had personal responsibility for the care of one or more children.

The respondents were primarily Caucasian $(94 \%)$ with a mean grade point average of 3.33 $(\mathrm{S} . \mathrm{D} .=.50, \mathrm{Median}=3.40)$. One respondent was not included in these grade point average calculations, as the response given was 'yes'. Participants, on average, watched television in the evenings for 1.67 hours.

\section{Results}

The number of respondents completing all stages of the study was 54 yielding a reponse rate of $47 \%$. Fourteen respondents completed the initial survey, but were not present to complete the follow up surveys. This represents a $21 \%$ attrition rate. Additionally, three respondents of the initial survey failed to include their initial three day sleep recalls.

If students failed to fill in the necessary sleep recall information $(n=3)$, or if they did not complete the post study survey $(n=14)$, they were not included in the final data. Additionally there were two outliers who reported extreme pre-sleep times. Their sleep recalls for the three days prior to initial survey were 47.00 and 53.75 hours. As a comparison, the respondent with the third and next highest initial sleep recall total was 28.00. The differences between these highest two totals and the third highest are 19 and 25.75 hours, respectively. The mean total sleep of all other respondents on their pretest survey was 21.61. These two slept roughly twice the average amount of the remainder of the respondents. It was decided that these individuals had significant sleep issues that were outside of the scope of this study, and therefore their data are not included in the following analyses.

Repeated measures analysis of variance was utilized with one within-subjects factor, and two between-subjects factors. The within subjects factor was pretest compared to posttest, including both three day sleep recalls, as well as the ESS total scores. The between subjects factors were first, the participation in or conversely non participation in sleep education, and second, the keeping or not keeping of a sleep log for two weeks, both of which occurred in the selected groups in the interval between the pretests and posttests. The data were analyzed using SPSS for Windows version 14.0.0.

\section{Results}

\section{ESS Analysis}

Upon statistical analysis, there were no significant main effects or interactions with respect to ESS scores. Therefore, the focus of the following analysis will be on sleep times 
which did yield statistically significant effects. Additionally, the reliability coefficient $(\mathrm{a}=.583)$ for this ESS sample was relatively low. Therefore, given to the same sample under different circumstances, the sample may produce different results. Further analyses revealed deletion of the first ESS question would have increased the Alpha to .627, bringing it closer to a desired level. In comparison, initial ESS assessment research by Johns (1992) yielded a Chronbach's Alpha of .88 with highly correlated pretest-posttest scores $(r=.82)$.

\section{Sleep Times Analysis}

Due to group sizes which were less than 30 (except for the total number of those who received sleep education), tests to determine normality of the data were performed. Box's M Test showed that an acceptable degree homoscedasticity was present in the covariance structure (Box's $\mathrm{M}=9.003, \mathrm{~F}=.914$, sig. $=.512$ ). Levine's Test also showed that the error variance was relatively equal across the groups of subjects studied (Pretest Sleep: $\mathrm{F}=.824$, sig. $=$ .487 ; Posttest Sleep: $\mathrm{F}=.846$, sig. $=.475$ ).

The standardized residual for the time recorded on the pre survey as calculated with the Kolmogorov-Smirnov test (with Lilliefors Significance Correction) for normality was .111 $($ sig. $=.153)$, but for the post survey was .126 (sig.=.040). While these outcomes are not ideal, they do show normality in the pretest data. The Shapiro-Wilk's test of normality, which is shown to have more power for detecting nonnormality than the Kolmogorov-Smirnov test, yielded statistics of .928 (sig. $=.004)$ for the pretest and $.948(\mathrm{sig} .=.024)$ for the posttest, again indicating that there was lack of normality in the data. Further examination of the moment statistics indicates significant negative skewness and positive kurtosis in the pre-sleep time residuals and significant negative skewness in the post-sleep time residuals. This negative skewness is a reflection of the greater range of sleeping times in undersleepers, as compared to oversleepers. Since the cell sample sizes were similar, and there were no outliers with standardized residuals more than three standard deviations away from the mean, the ANOVA test was fairly robust to these violations. However, one should view the p-values as approximate rather than exact.

Both pretest and posttest sleep times for all subjects were negatively skewed. It was more likely that an outlier would be someone who got less sleep than it was for an outlier to get an equivalent amount of greater sleep. Restated, there is a greater degree of variation in getting less than average sleep than there is in getting more than average sleep. The minimum standardized residual for the pretest sleep times was -2.80 , and for the posttest sleep times was 2.69. There is great variance in the amounts of sleep that subjects received, especially when getting less than an average amount of sleep.

Because the study involved four groups, a Bonferroni Correction was applied to p-values, once for each pairwise comparison (then the resultant $p$ values were multiplied by four).

The group that received sleep education and completed a sleep log reported an improved sleep recall by 2.68 hours over the previous three nights. This is the equivalent of nearly 54 minutes of sleep per night.

Students recruited to participate in this study received, on average, just over seven hours of sleep per night. This represents a decline in sleep of nearly an hour from their 1960s counterparts (Hicks \& Pellegrini 1991). Additionally, it is less than what is collectively needed to be at the highest physical, mental and emotional capabilities.

Alone, recording sleep amounts or sleep education did not improve the amount of sleep nor was a reduction of tiredness demonstrated. However, when combined, results indicated students significantly increased sleep times (50 minutes per night). As with the other groups, no reduction of tiredness was observed. 
Table 3

Tests of Within-Subjects Effects (with Bonferroni Correction)

\begin{tabular}{|l|c|c|c|c|c|c|}
\hline \multicolumn{1}{|c|}{ Source } & n & $\begin{array}{c}\text { Type III Sum } \\
\text { of Squares }\end{array}$ & df & $\begin{array}{c}\text { Mean } \\
\text { Square }\end{array}$ & F & Sig. \\
\hline Pre vs. Post Test & 17 & 2.607 & 1 & 2.607 & 0.978 & 0.328 \\
\hline $\begin{array}{l}\text { Pre vs. Post Test } \\
\text { * Sleep Education }\end{array}$ & 9 & 5.460 & 1 & 5.460 & 2.049 & 0.159 \\
\hline $\begin{array}{l}\text { Pre vs. Post Test } \\
\text { * Sleep Log }\end{array}$ & 17 & 2.923 & 1 & 2.923 & 1.097 & 0.300 \\
\hline $\begin{array}{l}\text { Pre vs. Post Test } \\
\text { * Education Log }\end{array}$ & 9 & 53.606 & 1 & 53.606 & 20.118 & $4.54 \mathrm{E}-05$ \\
\hline Error (Pre-Post) & & 127.901 & 48 & 2.665 & & \\
\hline
\end{tabular}

Table 4

Sleep Times, Group's Pretest - Posttest Differences

\begin{tabular}{|c|c|c|c|c|}
\hline Group & $\log$ & Education & Test & Mean \\
\hline & \multirow{3}{*}{ No Log Completed } & \multirow{3}{*}{ No Sleep Education } & Pretest & 20.38 \\
\hline \multirow[t]{2}{*}{ A } & & & Posttest & 21.39 \\
\hline & & & Difference & 1.01 \\
\hline & \multirow{3}{*}{ No Log Completed } & \multirow{3}{*}{ Received Sleep Education } & Pretest & 22.86 \\
\hline \multirow[t]{2}{*}{$\mathrm{B}$} & & & Posttest & 21.56 \\
\hline & & & Difference & -1.31 \\
\hline \multirow{3}{*}{$\mathrm{C}$} & \multirow{3}{*}{ Logged } & \multirow{3}{*}{ No Sleep Education } & Pretest & 22.08 \\
\hline & & & Posttest & 21.03 \\
\hline & & & Difference & -1.05 \\
\hline \multirow{3}{*}{$\mathrm{D}$} & \multirow{3}{*}{ Logged } & \multirow{3}{*}{ Received Sleep Education } & Pretest & 21.80 \\
\hline & & & Posttest & 24.47 \\
\hline & & & Difference & 2.68 \\
\hline
\end{tabular}

\section{Discussion}

\section{Limitations to the Statistical Analyses}

There are some limitations to the preceding statistical analyses. Although classes were randomly assigned to treatments, the groups were not randomly formed. Therefore, there may have been confounding issues such as different instructors from one group to another and different times for individual sections of the personal health course. Exposure to diverse instructors and environments may account for some of the demonstrable differences, but having a pre-test/post-test model could have been effective in countering this factor. Ultimately, it was change of sleep times within each group that was measured, and not merely the difference between groups total sleep times.

Also, as stated previously, there was nonagreement of Kolmogorov-Smirnov and Shapiro-Wilk tests for normality. The ShapiroWilk test was not conclusive for either the pretest or the posttest, but it is more sensitive to the outlying ends of the data set, and there was great variance on the amounts of sleep people reported (especially on the below average left tail). Stated another way, the difference between the median amount of average nightly sleep per respondent (7.52 hours) and the lowest nightly average (3.72 hours) was 3.80 hours. On the 
other hand the difference between the median (again, 7.52 hours) and the highest average of nightly sleep (9.33 hours) was only 1.81 hours. The distance from the mean for the low end of data was over twice as long as the distance of the high end of the data. This demonstrates that sleep studies generally will need greater sample sizes than the present study to have a normal set of data from which conclusions can be drawn.

Finally, two respondents who reported pretest sleep times that were double the mean were excluded from the analysis, as it seems clear that they had significant sleep issues that were outside the scope of the research at hand.

\section{Limitations}

There are a number of limitations to this research. All data obtained was self reported data, and thus has some degree of subjectivity injected. The convenience sample obtained put students with similar schedules, and perhaps teacher preferences, in the same groups. The participants were overwhelmingly Caucasian, and primarily female. Generalizability is not possible beyond the study sample due to lack of random selection and random assignment to control and treatment groups. Additionally, because of the limited scope of this study, causation cannot be applied to the broader population.

Another limitation to this research lies in the fact that while initially it was planned to have the pre-test/post-test interval at exactly two weeks, one group's class was cancelled at the two week point due to the teacher scheduling an online component, and was further cancelled at the three week point due to weather considerations. These factors prevented the post data from being gathered until a full four weeks after the initial visit. Another instructor cancelled class due to illness at the two week point, and while roughly half of the post surveys were collected at that time, the other half had to be collected at three weeks.

\section{Conclusions and Recommendations for Further Study}

It is interesting to note that those groups which received only one intervention showed, on average, decreased sleep times. This suggests improvement in sleep times is more likely when students learn and actively track their behavior. Instructors who seek to not only increase the knowledge of their students, but to also increase the likelihood that students will improve in their regular health behaviors, may want to consider implementing logging or journaling activities with their instruction.

This study demonstrated that when sleep education contained both an instruction factor and a logging factor, a student was more likely to improve sleep. Future research including a larger set of participants would be much more effective in evaluating these factors. The data sets tend to be skewed, as it is more common for students to undersleep, and to do so with greater variation, than it is for them to oversleep. Much of the previous work in this subject involves students in introductory college psychology courses. This would likely facilitate larger participant numbers, as introductory psychology is a prerequisite for many majors. It may also provide a more diverse set of subjects.

Using the ESS provided no meaningful data for this research. This may have been because the timing of many posttest surveys was delayed. Perhaps the use of the profile of mood states (POMS) as developed by McNair, Lorr, \& Droppleman (1971) might more accurately reflect the improvement obtained through additional sleep education and keeping a sleep $\log$. While this instrument is more cumbersome, it may be worth the effort required for its use.

Although logistically more difficult during the ten to fifteen minutes of sleep education, it would be more effective, from a research point of view, to randomly assign students to different groups within classes. Those participants not receiving sleep education could be given a brief, but useful, presentation on an item unrelated to sleep in an adjacent room.

There clearly is potential for reduced sleep due to the increased use of technological devices such as cell phones, mp3 players and computers, which all are becoming more available and integral in the lives of college students. No 
research article relating to this factor was found. Perhaps this is because researchers are hesitant to suggest interventions relating to devices that are quickly entrenching themselves into the lives of people generally, and especially the young. Hopefully someone will soon be brave enough to assess the issue and recommend changes in practice, if needed.

This study was concerned solely with sleep habits and tiredness. The use of a log could be implemented in most phases of health education. Measuring outcomes will likely yield greater results in most health education endeavors, rather than merely discussing them.

While the evaluation of tiredness is important in addressing the lack of necessary sleep, more research evaluating the emotional state of persons relative to their sleep would be helpful. If this truly is where the greatest toll is taken on individuals who are lacking in sleep, then showing the effect it has on interpersonal relationships and in a person's overall sense of well-being may allow a greater general emphasis on this problem.

\section{References}

Alapin, I., Fichten C. S., Libman E., Creti, L., Bailes, S., \& Wright, J. (2000). How is good and poor sleep in older adults and college students related to daytime sleepiness, fatigue, and ability to concentrate? Journal of Psychosomatic Research, 49, 381-390.

Allgower, A., Wardle, J., \& Steptoe, A. (2001). Depressive symptoms, social support, and personal health behaviors in young men and women. Health Psychology, 20, 223-227.

Buboltz, W. C., Brown, F., \& Soper, B. (2001). Sleep habits and patterns of college students: A preliminary study. Journal of American College Health, 50(3), 131-135.

Capriccioso, R. (2006, June 15). Sleepy hollow. Inside Higher Ed. Retrieved March 24, 2007, from http://www.insidehighered.com/news/2006/06/15/sleep

Coren, S. (1994). The prevalence of self-reported sleep disturbances in young adults. The International Journal of Neuroscience, 79(1-2), 67-73.

Dennis, C. L., Ross, L. (2005). Relationships among infant sleep patterns, maternal fatigue, and development of depressive symptomatology. Birth, 32, 187-193.

Engle-Friedman, M., Riela, S., Golan, R., Ventuneac, A. M., Davis, C. M., Jefferson, A. D. et al. (2003). The effect of sleep loss on next day effort. Journal of Sleep Research, 12, 113-124.

Giesecke, M. E. (1987). The symptom of insomnia in university students. Journal of American College Health, 35, 215-221.

Hicks, R. A., Fernandez, C., \& Pellegrini R. J. (2001a). Self-reported sleep durations of college students: normative data for 1978-79, 1988-89, and 2000-01. Perceptual and Motor Skills, 93, 139-140.

Hicks, R. A., Fernandez, C., \& Pellegrini R. J. (2001b). Striking changes in the sleep satisfaction of university participants over the last two decades. Perceptual and Motor Skills, 93, 660.

Hicks, R. A., \& Pellegrini, R. J. (1991). The changing sleep habits of college students. Perceptual and Motor Skills, 72(3 Pt 2), 1106.

Howell, A. J., Jahrig, J. C., and Powell, R. A. (2004). Sleep quality, sleep propensity and academic performance. Perceptual and Motor Skills, 99, 525-535.

Jindal, R. D., \& Thase, M. E. (2004). Treatment of insomnia associated with clinical depression. Sleep Medicine Reviews, 8, 19-30.

Johns, M. W. (1991). A new method for measuring daytime sleepiness: the Epworth sleepiness scale. Sleep, 14, 540-545.

Kohatsu, N. D., Tsai, R., Young, T., VanGilder, R., Burmeister, L. F., Stromquist, A. M. et al. (2006). Sleep duration and body mass index in a rural population. Archives of Internal Medicine, 166, 1701-1705.

Lack, L. C. (1986). Delayed sleep and sleep loss in university students. Journal of American College Health, 35(3), 105-110.

McNair, D. M., Lorr, M., \& Droppleman L. F. (1971). Profile of Mood States: Manual. San Diego, CA: Education and Industrial Testing Service. 
Meltzer, L. J., \& Mindell, J. A. (2007). Relationship between child sleep disturbances and maternal sleep, mood, and parenting stress: A pilot study. Journal of Family Psychology, 21, 67-73.

National Sleep Foundation (2005). Students are falling deeply in debt - sleep debt that is! Sleepmatters 7(3). Retrieved April 3, 2007, from http://www.sleepfoundation.org/site/c.huIXKjM0IxF/b.2422493/k.59A6/Students are Falling_D eeply in Debt Sleep Debt That Is.htm

Peppard, P. E., Szklo-Coxe, M., Hla, M., \& Young, T. (2006). Longitudinal association of sleep-related breathing disorder and depression. Archives of Internal Medicine 166, 1709-1715.

Pilcher J. J., Ginter, D. R., \& Sadowsky, B. (1997). Sleep quality verses sleep quantity: relationships between sleep and measures of health, well-being, and sleepiness in college students. Journal of Psychosomatic Research, 42, 583-596.

SPSS, Inc. (2005). SPSS for Windows, Rel. 14.0.0, 2005. Chicago: Author.

Steptoe, A., Peacey, V., \& Wardle J. (2006). Sleep duration and health in young adults. Archives of Internal Medicine, 166, 1689-1692.

\author{
$\underline{\text { Author Information }}$ \\ Daniel J. Prestwich, MHE (ISU graduate) \\ Idaho State University \\ 766 North University \\ Blackfoot, ID 83221 \\ E-Mail: dkprest@msn.com \\ Linda L. Rankin, PhD, RD, FADA* \\ Professor \\ Idaho State University \\ Department of Health and Nutrition Sciences \\ Stop 8109 \\ Pocatello, ID 83209-8109 \\ E-Mail: ranklind@isu.edu \\ Jeff Housman, PhD, CHES \\ Assistant Professor \\ Idaho State University \\ Department of Health and Nutrition Sciences \\ Stop 8109 \\ Pocatello, ID 83209-8109 \\ E-Mail: housjeff@isu.edu \\ * corresponding author
}

